\title{
ON THE CARLESON MEASURE CHARACTERIZATION OF BMO FUNCTIONS ON THE UNIT SPHERE
}

\author{
MIROLJUB JEVTIĆ \\ (Communicated by Clifford J. Earle)
}

\begin{abstract}
A higher dimensional version of the well-known Carleson measure characterization of BMO functions is given.
\end{abstract}

\section{INTRODUCTION}

The main purpose of this paper is to prove a higher dimensional version of the well-known Carleson measure characterization of BMO functions.

Let $B$ denote the unit ball in $C^{n}, n \geq 1$, and $m$ the $2 n$-dimensional Lebesgue measure on $B$ normalized so that $m(B)=1$, while $\sigma$ is the normalized surface measure on its boundary $S$. For the most part we will follow the notation and terminology of Rudin [9].

For $\xi \in S$ and $0<\delta \leq 2$, put $Q_{\delta}(\xi)=\{\eta \in S:|1-\langle\eta, \xi\rangle|<\delta\}$.

The class BMO consists of functions $f \in L^{2}(\sigma)$ for which

$$
\|f\|_{\mathrm{BMO}}^{2}=\sup \frac{1}{\sigma(Q)} \int_{Q}\left|f(\xi)-f_{Q}\right|^{2} d \sigma(\xi)<\infty,
$$

where $f_{Q}$ denotes the averages of $f$ over $Q$ and the supremum is taken over all $Q=Q_{\delta}(\xi)$.

A positive Borel measure $\mu$ on $B$ is called a Carleson measure if $\mu\left(B_{\delta}(\xi)\right) \leq$ $C \delta^{n}$, for all $\xi \in S, 0<\delta \leq 2$, where $B_{\delta}(\xi)=\{z \in B:|1-\langle z, \xi\rangle|<\delta\}$.

Here and elsewhere constants are denoted by $C$, which may indicate a different constant from one occurrence to the next.

For $f \in C^{1}(B), D f=\left(\frac{\partial f}{\partial z_{1}}, \ldots, \frac{\partial f}{\partial z_{n}}\right)$ denotes the complex gradient of $f$, $\nabla f=\left(\frac{\partial f}{\partial x_{1}}, \ldots, \frac{\partial f}{\partial x_{2 n}}\right), z_{k}=x_{2 k-1}+i x_{2 k}, k=1,2, \ldots, n$, denotes the real gradient of $f$ and let

$$
\left|\nabla_{T} f(z)\right|^{2}=2\left(|D f(z)|^{2}-|R f(z)|^{2}+|D \bar{f}(z)|^{2}-|R \bar{f}(z)|^{2}\right)
$$

be the tangential gradient of $f$. As usual, $R$ denotes the radial derivative $R=\sum_{j=1}^{n} z_{j} \frac{\partial}{\partial z_{j}}$.

Received by the editors September 23, 1992 and, in revised form, April 14, 1994.

1991 Mathematics Subject Classification. Primary 32A37.

Key words and phrases. Carleson measures, BMO spaces. 
For $\psi \in L^{1}(\sigma), P[\psi]$ denotes the Poisson-Szegö integral defined for $z \in$ $B$ by $P[\psi](z)=\int_{S} P(z, \xi) \psi(\xi) d \sigma(\xi)$, where $P(z, \xi)=\left(\frac{1-|z|^{2}}{|1-\langle z, \xi\rangle|^{2}}\right)^{n}$ is the Poisson-Szegő kernel for $B$.

Theorem. Let $f \in L^{2}(\sigma)$ and $F=P[f]$. Then the following statements are equivalent:

(a) $f \in \mathrm{BMO}$.

(b) $\quad d \mu(z)=|\nabla F(z)|^{2}\left(1-|z|^{2}\right) d m(z)$ is a Carleson measure.

(c) $\quad d \nu(z)=\left|\nabla_{T} F(z)\right|^{2} d m(z)$ is a Carleson measure.

If $n=1$, then the Theorem is well known (see, for example, [10] ). Note that $d \mu(z)=d \nu(z)$ in this case. See also [2].

Recall that $H^{2}$ is a subspace of $L^{2}(\sigma)$ consisting of functions $f$ such that $P[f]$ is holomorphic in $B$. If $f \in H^{2}, n>1$, the equivalence of statements (a) and (b) was proved in [5] and the equivalence (a) $\Longleftrightarrow$ (c) in [3].

\section{Preliminaries}

As in [9], we say that a function $u \in C^{2}(B)$ is $\mathscr{M}$-harmonic in $B, u \in \mathscr{M}$, if $\widetilde{\Delta} u(z)=0$ for every $z \in B$. The operator $\tilde{\Delta}$ is the invariant Laplacian defined by $\widetilde{\Delta} u(z)=\Delta\left(u \circ \varphi_{z}\right)(0), z \in B$, where $\Delta$ is the ordinary Laplacian and $\varphi_{z}$ the standard automorphism of $B$ taking 0 to $z$ (see [9]).

For $a \in B$ and $0<r<1$ let $E_{r}(a)=\left\{z \in B:\left|\varphi_{a}(z)\right|<r\right\}$. The measure $\tau$ defined on $B$ by $d \tau(z)=\left(1-|z|^{2}\right)^{-n-1} d m(z)$ is $M$-invariant (see [9]).

Lemma 2.1 ([3], [8]). If $f \in L^{2}(\sigma)$, then

$$
\int_{B} G(z) \widetilde{\Delta}|P[f]|^{2}(z) d \tau(z)=\int_{S}|f(\xi)-P[f](0)|^{2} d \sigma(\xi),
$$

where $G(z)=\frac{1}{2 n} \int_{|z|}^{1} t^{1-2 n}\left(1-t^{2}\right)^{n-1} d t, \quad z \in B \backslash\{0\}$.

Lemma $2.2([7])$. Let $F=P[f], f \in L^{2}(\sigma)$. Then

$$
\begin{aligned}
|z|^{2}\left|\nabla_{T} F(z)\right|^{2}=2 & \left(\left(1-|z|^{2}\right)\left(|R F(z)|^{2}+|R \bar{F}(z)|^{2}\right)\right. \\
& \left.+\sum_{i<j}\left|T_{i j} F(z)\right|^{2}+\sum_{i<j}\left|T_{i j} \bar{F}(z)\right|^{2}\right),
\end{aligned}
$$

where $T_{i j}=\bar{z}_{i} \frac{\partial}{\partial z_{j}}-\bar{z}_{j} \frac{\partial}{\partial z_{i}}$ are tangential derivatives.

Lemma 2.3 ([7]). Let $1 \leq i<j \leq n, 1 \leq k \leq n, 0<r<1$. There is a constant $K$ such that if $f \in \mathscr{M}$, then

$$
\left|T_{i j} u_{k}(w)\right| \leq K\left(1-|w|^{2}\right)^{-1 / 2} \int_{E_{r}(w)}\left|u_{k}(z)\right| d \tau(z), \quad w \in B
$$

where $u_{k}=\frac{\partial f}{\partial z_{k}}$ or $u_{k}=\frac{\partial f}{\partial \bar{z}_{k}}$.

Remark. In [7] analogous estimates for $T_{i j} R f$ and $T_{i j} \overline{R \bar{f}}$, based on the reproducing formulas which are derived in [1], are obtained. Since the same reproducing formulas holds for the functions $u_{k}$ (see [1]), the same argument 
can be applied to derive the estimate (2.3). Similarly, one can conclude that (2.4)

$$
\left|T_{i j} f(w)\right| \leq K\left(1-|w|^{2}\right)^{-1 / 2} \int_{E_{r}(w)}|f(z)| d \tau(z), \quad w \in B, 1 \leq i<j \leq n .
$$

Lemma 2.4. Let $f \in L^{2}(\sigma)$ and $F=P[f]$. Then

$$
\int_{B} G(w) \tilde{\Delta}|F|^{2}(w) d \tau(w) \leq C \int_{B}\left(1-|w|^{2}\right)^{n} \tilde{\Delta}|F|^{2}(w) d \tau(w)
$$

Proof. For $|z|>1 / 4$, we have $G(z) \leq 16^{n}\left(1-|z|^{2}\right)^{n}$.

We may proceed as in the proof of Lemma 2.1 ([7]) to conclude that $|R F(z)|^{2},|R \bar{F}(z)|^{2},\left|T_{i j} F(z)\right|^{2}$ and $\left|T_{i j} \bar{F}(z)\right|^{2}, 1 \leq i<j \leq n$, have $\mathscr{M}$ subharmonic behaviour. Hence, $\tilde{\Delta}|F|^{2}(z)$ has $\mathscr{M}$-subharmonic behaviour by (2.2). Thus, for $|z| \leq 1 / 4$, we have

$$
\tilde{\Delta}|F|^{2}(z) \leq C \int_{E_{1 / 4}(z)} \tilde{\Delta}|F|^{2}(w) d \tau(w) \leq C \int_{\frac{1}{2} B} \tilde{\Delta}|F|^{2}(w)\left(1-|w|^{2}\right)^{n} d \tau(w),
$$

and therefore

$$
\int_{|z| \leq 1 / 4} G(z) \tilde{\Delta}|F|^{2}(z) d \tau(z) \leq C \int_{B}\left(1-|w|^{2}\right)^{n} \tilde{\Delta}|F|^{2}(w) d \tau(w)
$$

\section{Proof of THE TheOREM}

The proof of Theorem 1 ([5]) shows that if $f \in \mathrm{BMO}$, then $d \mu(z)$ is a Carleson measure.

(b) $\Longrightarrow$ (c) It is easy to see that if $d \mu(z)$ is a Carleson measure, then $\left(1-|z|^{2}\right)|R F(z)|^{2} d m(z)$ and $\left(1-|z|^{2}\right)|R \bar{F}(z)|^{2} d m(z)$ are Carleson measures. Hence, by Lemma 2.2, to show that $d \nu(z)$ is a Carleson measure it suffices to show that $\left|T_{i j} F(z)\right|^{2} d m(z)$ and $\left|T_{i j} \bar{F}(z)\right|^{2} d m(z)$ are Carleson measures for all $1 \leq i<j \leq n$.

We will show that $\left|T_{i j} F(z)\right|^{2} d m(z)$ is a Carleson measure. Analogously, we may prove that $\left|T_{i j} \bar{F}(z)\right|^{2} d m(z)$ is a Carleson measure.

An integration by parts shows that

$$
F(z)=\int_{0}^{1}\{R F(t z)+\overline{R \bar{F}}(t z)+F(t z)\} d t
$$

From this we conclude that it is sufficient to prove that

$$
\begin{gathered}
{\left[\int_{0}^{1}\left|T_{i j} \frac{\partial F}{\partial z_{k}}(t z)\right| d t\right]^{2} d m(z),} \\
{\left[\int_{0}^{1}\left|T_{i j} \frac{\partial F}{\partial \overline{z_{k}}}(t z)\right| d t\right]^{2} d m(z) \text { and }\left[\int_{0}^{1}\left|T_{i j} F(t z)\right| d t\right]^{2} d m(z)}
\end{gathered}
$$

are Carleson measures for all $1 \leq k \leq n$. Let $u_{k}=\frac{\partial F}{\partial z_{k}}$ or $u_{k}=\frac{\partial F}{\partial \overline{z_{k}}}$. 
Using Lemma 2.3 and the fact that $1-|w|^{2} \cong|1-\langle z, w\rangle| \cong 1-|z|^{2}$, for $w \in E_{r}(z)$, we see that for any $s>0$

$$
\begin{aligned}
\int_{0}^{1}\left|T_{i j} u_{k}(t w)\right| d t & \leq C \int_{0}^{1}\left[\int_{E_{r}(t w)} \frac{\left(1-|z|^{2}\right)^{s}\left|u_{k}(z)\right|}{|1-t\langle w, z\rangle|^{n+s+3 / 2}} d m(z)\right] d t \\
& \leq C \int_{0}^{1}\left[\int_{B} \frac{\left(1-|z|^{2}\right)^{s}}{|1-t\langle w, z\rangle|^{n+s+3 / 2}}\left|u_{k}(z)\right| d m(z)\right] d t \\
& \leq C \int_{B} \frac{\left(1-|z|^{2}\right)^{s}\left|u_{k}(z)\right|}{|1-\langle w, z\rangle|^{n+s+1 / 2}} d m(z) .
\end{aligned}
$$

Now we proceed as in [6]. Let $\xi \in S$ and $\delta>0$. By standard estimates (see [9], p. 17)

$$
\int_{B} \frac{\left(1-|z|^{2}\right)^{s-1 / 4}}{|1-\langle w, z\rangle|^{n+s+5 / 4}} d m(z) \leq C\left(1-|w|^{2}\right)^{-1 / 2} .
$$

Thus by Hölders inequality

$$
\begin{aligned}
\int_{B_{\delta}(\xi)} & {\left[\int_{0}^{1}\left|T_{i j} u_{k}(t w)\right| d t\right]^{2} d m(w) } \\
\leq & C \int_{B_{\delta}(\xi)}\left[\int_{B} \frac{\left(1-|z|^{2}\right)^{s}\left|u_{k}(z)\right|}{|1-\langle z, w\rangle|^{n+s+1 / 2}} d m(z)\right]^{2} d m(w) \\
\leq & C \int_{B_{\delta}(\xi)}\left[\int_{B} \frac{\left|u_{k}(z)\right|^{2}\left(1-|z|^{2}\right)^{s+1 / 4}}{|1-\langle z, w\rangle|^{n+s-1 / 4}} d m(z)\right. \\
& \left.\times \int_{B} \frac{\left(1-|z|^{2}\right)^{s-1 / 4}}{|1-\langle z, w\rangle|^{n+s+5 / 4}} d m(z)\right] d m(w) \\
\leq & C \int_{B_{\delta}(\xi)}\left[\int_{B} \frac{\left|u_{k}(z)\right|^{2}\left(1-|z|^{2}\right)^{s+1 / 4}}{|1-\langle z, w\rangle|^{n+s-1 / 4}} d m(z)\right]\left(1-|w|^{2}\right)^{-1 / 2} d m(w) \\
= & C \int_{B_{\delta}(\xi)}\left[\int_{B_{2 \delta}(\xi)} \frac{\left|u_{k}(z)\right|^{2}\left(1-|z|^{2}\right)^{s+1 / 4}}{|1-\langle z, w\rangle|^{n+s-1 / 4}} d m(z)\right]\left(1-|w|^{2}\right)^{-1 / 2} d m(w) \\
& +C \int_{B_{\delta}(\xi)}\left[\sum_{j} \int_{A_{j}} \frac{\left|u_{k}(z)\right|^{2}\left(1-|z|^{2}\right)^{s+1 / 4}}{|1-\langle z, w\rangle|^{n+s-1 / 4}} d m(z)\right]\left(1-|w|^{2}\right)^{-1 / 2} d m(w) \\
= & I_{1}+I_{2},
\end{aligned}
$$

where $A_{j}=\left\{z \in B: 2^{j} \delta \leq|1-\langle z, \xi\rangle|<2^{j+1} \delta\right\}, \quad j=1,2, \ldots$ As above we have

$$
\int_{B} \frac{\left(1-|w|^{2}\right)^{-1 / 2}}{|1-\langle z, w\rangle|^{n+s-1 / 4}} d m(w) \leq C\left(1-|z|^{2}\right)^{3 / 4-s}
$$

(we may suppose that $s>3 / 4$ ). 
Hence by Fubini's theorem

$$
\begin{aligned}
I_{1} & \leq C \int_{B_{2 \delta}(\xi)}\left|u_{k}(z)\right|^{2}\left(1-|z|^{2}\right)^{s+1 / 4}\left[\int_{B} \frac{\left(1-|w|^{2}\right)^{-1 / 2}}{|1-\langle z, w\rangle|^{n+s-1 / 4}} d m(w)\right] d m(z) \\
& \leq C \int_{B_{2 \delta}(\xi)}\left(1-|z|^{2}\right)\left|u_{k}(z)\right|^{2} d m(z) \leq C \delta^{n}
\end{aligned}
$$

since, from the assumption that $\left(1-|z|^{2}\right)|\nabla F(z)|^{2} d m(z)$ is a Carleson measure, it follows easily that $\left(1-|z|^{2}\right)\left|u_{k}(z)\right|^{2} d m(z)$ is a Carleson measure for all $1 \leq k \leq n$.

Notice that if $w \in B_{\delta}(\xi)$ and $z \in A_{j}$ we have

$$
|1-\langle w, z\rangle|^{1 / 2} \geq|1-\langle z, \xi\rangle|^{1 / 2}-|1-\langle w, \xi\rangle|^{1 / 2} \geq \frac{1}{2}(\sqrt{2}-1) 2^{j / 2} \delta^{1 / 2}
$$

Hence

$$
I_{2} \leq C \sum_{j}\left(2^{j} \delta\right)^{-n-1 / 2} \int_{A_{j}}\left|u_{k}(z)\right|^{2}\left(1-|z|^{2}\right) d m(z) \int_{B_{\delta}(\xi)}\left(1-|w|^{2}\right)^{-1 / 2} d m(w) .
$$

From the estimate $\sigma(\{\eta \in S:|1-r\langle\eta, \xi\rangle|<\delta\}) \leq C \delta^{n}, 0 \leq r<1$ (see [9], p.67), it follows easily that $\int_{B_{\delta}(\xi)}\left(1-|w|^{2}\right)^{-1 / 2} d m(w) \leq C \delta^{n+1 / 2}$. Using this and again the fact that $\left|u_{k}(z)\right|^{2}\left(1-|z|^{2}\right) d m(z)$ is a Carleson measure we get $I_{2} \leq C \delta^{n}$.

Now we will show that $\left(1-|w|^{2}\right)|F(w)|^{2} d m(w)$ is also a Carleson measure. Without loss of generality we may assume that $F(0)=0$. Let $s>1$ and $0<r<1$. Using Lemma 3.1 in [7] we find that

$$
\begin{aligned}
|F(w)|^{2} & =\left|\int_{0}^{1} \frac{d}{d t} F(t w) d t\right|^{2} \leq \int_{0}^{1}|\nabla F(t w)|^{2} d t \\
& \leq C \int_{0}^{1}\left(\int_{E_{r}(t w)} \frac{|\nabla F(z)|^{2}\left(1-|z|^{2}\right)^{s} d m(z)}{|1-\langle z, t w\rangle|^{n+1+s}}\right) d t \\
& \leq C \int_{0}^{1}\left(\int_{B} \frac{|\nabla F(z)|^{2}\left(1-|z|^{2}\right)^{s} d m(z)}{|1-t\langle z, w\rangle|^{n+1+s}}\right) d t \\
& \leq C \int_{B} \frac{|\nabla F(z)|^{2}\left(1-|z|^{2}\right)^{s} d m(z)}{|1-\langle z, w\rangle|^{n+1+s}} .
\end{aligned}
$$

Here we have used the fact that $|1-\langle z, t w\rangle| \cong 1-|z|^{2}$, for $z \in E_{r}(t w)$, and the simple estimate $|1-t\langle z, w\rangle|^{-1} \leq 2|1-\langle z, w\rangle|^{-1}, 0<t<1, z, w \in B$. (In fact, by an integration we can get a better estimate.) 
Thus, for $\xi \in S$ and $\delta>0$ we have

$$
\begin{aligned}
\int_{B_{\delta}(\xi)}(1- & \left.|w|^{2}\right)|F(w)|^{2} d m(w) \\
\leq & C \int_{B_{\delta}(\xi)}\left(1-|w|^{2}\right)\left(\int_{B_{2 \delta}(\xi)} \frac{|\nabla F(z)|^{2}\left(1-|z|^{2}\right)^{s} d m(z)}{|1-\langle z, w\rangle|^{n+1+s}}\right) d m(w) \\
& +C \int_{B_{\delta}(\xi)}\left(\sum_{j} \int_{A_{j}} \frac{|\nabla F(z)|^{2}\left(1-|z|^{2}\right)^{s} d m(z)}{|1-\langle z, w\rangle|^{n+1+s}}\right)\left(1-|w|^{2}\right) d m(w),
\end{aligned}
$$

where $A_{j}=\left\{z \in B: 2^{j} \delta \leq|1-\langle z, \xi\rangle|<2^{j+1} \delta\right\}, j=1,2, \ldots$.

Since $\left(1-|z|^{2}\right)|\nabla F(z)|^{2} d m(z)$ is a Carleson measure, as above, we find that $\int_{B_{\delta}(\xi)}\left(1-|w|^{2}\right)|F(w)|^{2} d m(w) \leq C \delta^{n}$.

Using this and the estimate (2.4) we conclude that $\left(\int_{0}^{1}\left|T_{i j} F(t z)\right|^{2} d t\right)^{2} d m(z)$ is a Carleson measure for all $1 \leq i, j \leq n$.

(c) $\Rightarrow($ a) Assume that $d \nu(z)$ is a Carleson measure. Then

$$
\sup _{z \in B} \int_{B} \frac{\left(1-|z|^{2}\right)^{n}}{|1-\langle w, z\rangle|^{2 n}} d \nu(w)<\infty
$$

(see [3]). To show that $f \in \mathrm{BMO}$ it suffices to show that the Garcia norm of $f$,

is finite.

$$
\|f\|_{G}^{2}=\sup _{z \in B} \int_{S}|f(\xi)-P[f](z)|^{2} P(z, \xi) d \sigma(\xi),
$$

By (2.1) and Lemma 2.4 we have

$$
\|f\|_{G}^{2} \leq C \sup _{z \in B} \int_{B}\left(1-|w|^{2}\right)^{n} \tilde{\Delta}\left|P\left[f \circ \varphi_{z}\right]\right|^{2}(w) d \tau(w) .
$$

Note by the $M$-invariance of $P, \tilde{\Delta}$ and $\tau$ that the last integral is equal to $\int_{B}\left(1-\left|\varphi_{z}(w)\right|^{2}\right)^{n} \tilde{\Delta}|P[f]|^{2}(w) d \tau(w)$, which is in turn equal to

$$
\int_{B} \frac{\left(1-|z|^{2}\right)^{n}\left(1-|w|^{2}\right)^{n}}{|1-\langle z, w\rangle|^{2 n}} \widetilde{\Delta}|P[f]|^{2}(w) d \tau(w),
$$

by Theorem 2.2.2 ([9], p. 26).

Using the formula for the invariant Laplacian given in Theorem 4.1.3 of Rudin's book [9] and the fact that $P[f]$ is an $\mathscr{M}$-harmonic function we find that $\widetilde{\Delta}|P[f]|^{2}(w)=2\left(1-|w|^{2}\right)\left|\nabla_{T} P[f](w)\right|^{2}$. Thus,

$$
\|f\|_{G}^{2} \leq C \sup _{z \in B} \int_{B} \frac{\left(1-|z|^{2}\right)^{n}}{|1-\langle z, w\rangle|^{2 n}} d \nu(w)<\infty .
$$

\section{REFERENCES}

1. P. Ahern and C. Cascante, Exceptional sets for Poisson integral of potentials on the unit sphere in $C^{n}, p \leq 1$, Pacific J. Math. 153 (1992), 1-15.

2. A. Baernstein II, Analytic functions of bounded mean oscilation, Aspects of Contemporary Complex Analysis, Academic Press, New York, 1980, pp. 3-36.

3. J. S. Choa and B. R. Choe, $A$ Littlewood-Paley type identity and a characterization of $B M O A$, Complex Variables Theory Appl. 17 (1991), 15-23. 
4. C. Fefferman and E. Stein, $H^{p}$ spaces of several variables, Acta Math. 129 (1972), 137-193.

5. M. Jevtić, A note on the Carleson measure characterization of BMOA functions on the unit ball, Complex Variables Theory Appl. 17 (1992), 189-194.

6. $\ldots$, On the Carleson measure characterization of BMOA functions on the unit ball, Proc. Amer. Math. Soc. 114 (1992), 379-386.

7. M. Jevtić and M. Pavlović, On $\mathscr{M}$-harmonic Bloch space, Proc. Amer. Math. Soc. 123 (1995), 1385-1392.

8. M. Pavlovic, Inequalities for the gradient of eigenfunctions of the invariant Laplacian in the unit ball, Indag. Math. (N.S.) 2 (1991), 89-98.

9. W. Rudin, Function theory in the unit ball of $C^{n}$, Springer-Verlag, New York, 1980.

10. K. Zhu, Operator theory in function spaces, Marcel Dekker, New York, 1990.

Matematički fakultet, Studentski TRg 16, 11000 Beograd, Yugoslavia 